\title{
Factors Predicting Sexual Dysfunction in Thai Cancer Patients after Treatment
}

\author{
Tiraporn Junda* and Bualuang Sumdaengrit RN \\ Faculty of Medicine Ramathibodi Hospital Mahidol University, Thailand
}

*Corresponding author: Tiraporn Junda, Nursing Instructor, Ramathibodi School of Nursing, Faculty of Medicine Ramathibodi Hospital Mahidol University, Bangkok, Thailand

submission: August 25, 2018; Published: October 04, 2018

\begin{abstract}
Aim: Purpose of this research was to study what factors that can predict sexual dysfunction in Thai patients with cancer after treatment.

Methods: This study was a predictive correlational research. Participants were 110 Thai patients with cancer of breast, prostate, bladder, and colon who were completed treatments from 6 months - 2 years from a university hospital, and 2 cancer specialize hospitals in 2013. The instruments were (1) the personal information and disease related treatment questionnaires (2) Dyadic Adjustment Scale developed by Spanier [1] and were translated into Thai by Kasemkitawatana (1993); and (3) the Sexual Health Dysfunction questionnaires developed by Kumdaeng [2]. Alpha Cronbach's coefficients were 89 both Dyadic Adjustment Scale and Sexual Health Dysfunction questionnaires. Data were analyzed by descriptive and multiple regression.
\end{abstract}

Result: Results found that gender, dyadic consensus, and affectional expression in the dyadic adjustment could predict the sexual dysfunction at 05 level, whereas age, duration of marriage, and other two subscales of dyadic adjustment could not predict the sexual dysfunction in patients with cancer after treatment.

Conclusion: Results confirmed that Thai cancer patients generally had to deal with sexual dysfunction. Female with low degree of dyadic consensus and less affectional expression predictively had more sexual dysfunction. These results can be used as evidence-based to provide and improve sexual health in patients with cancer after treatment.

Keywords: Sexual dysfunction; Thai cancer patients; Dyadic adjustment

\section{Introduction}

Sexual dysfunction is "the inability to express one's sexuality in a manner that is consistent with personal needs and preferences" [3]. In patients who has cancer in the organs involve sexual activity such as breast cancer, colon, rectum, prostrate, and bladder are vulnerable to this issue [4,5]. Sexual dysfunction may be caused by cancer itself or by the treatments. Those treatments were surgery, chemotherapy, or radiotherapy which potentially affect sexual organs and functions. These cancer patients suffer from loss or decreased function of the sexual organs. The prevalence of sexual dysfunction was about $20-100 \%$ [6] in patients with cancer. Cancer diagnosis and treatment highly impact on people's physical, psychosocial and social life. Up to date, cancer and symptom management usually are the central focus, however, Thai people still feel uncomfortable to express about sexual issues since it is a taboo topic [7]. As a result, sexual health problems in cancer patients have superficially been addressed or left to be untouched by the health care team.

In Thailand, breast cancer incident was reported to be the first cancer in Thai women [8-12]. From the reports of Thai National Cancer Institute of new breast cancer occurrence had been varied from $39.41-42.41 \%$ in 2012-2016. Similarly, several men with cancer of colon and rectum have been increasing to be the first to third cancer in Thai men [8-12]. With the data of Thai National Cancer Institute of new cancer occurrence had been reported from $13.67-17.21 \%$ in 2012-2016. These cancer patients generally spent a lot of effort to cope with their cancer diagnosis and managed the treatment's side effects until they were able to go through the difficult period of the treatments. In addition, they also had to confront with the difficulty of sexual dysfunction as a residual after the cancer treatments.

Even though nowadays cancer treatment has high effectiveness. After treatments, these cancer patients must adjust themselves before returning to their normal life since sexual function is affected from both cancer and treatments. If patients have strong marriage relationship and family adjustment or marital adjustment [13] with their partners before getting sick, they will have low sexual dysfunction $[14,15]$. Those cancer patients and their partners who have dyadic agreement, satisfaction, participate in activities together, and agreement with partner regarding emotional affection tend to be in a good relationship which helps improving a good sexual life after cancer treatment. In addition, sexual dysfunction is different between genders. From previous study, there was significant difference between genders of sexual dysfunction in 
patients with rectal cancer after surgery [16]. Male patients with rectal cancer had higher sexual dysfunction than female. However, there were also reported of female patients with breast cancer had more pain as well as less issue of relationship and love than male [17].

Other factors such as age [18-20] and duration of marriage [21] of cancer patients may affect sexual dysfunction. Patients who are older and had long duration of marriage might not be interfered with sexual dysfunction. Moreover, cancer patients with low dyadic adjustment may be exposed to more sexual dysfunction than those with high dyadic adjustment. These behaviors of cancer patients and their partners which are dyadic agreement, satisfaction, participate in activities together, and agreement with partner regarding emotional affection may be able to help reducing sexual dysfunction and increasing quality of life. To know what factors, influence the sexual dysfunction in cancer patients after treatment is necessary for health care providers to promote the sexual health in those cancer patients after treatment (Figure 1).

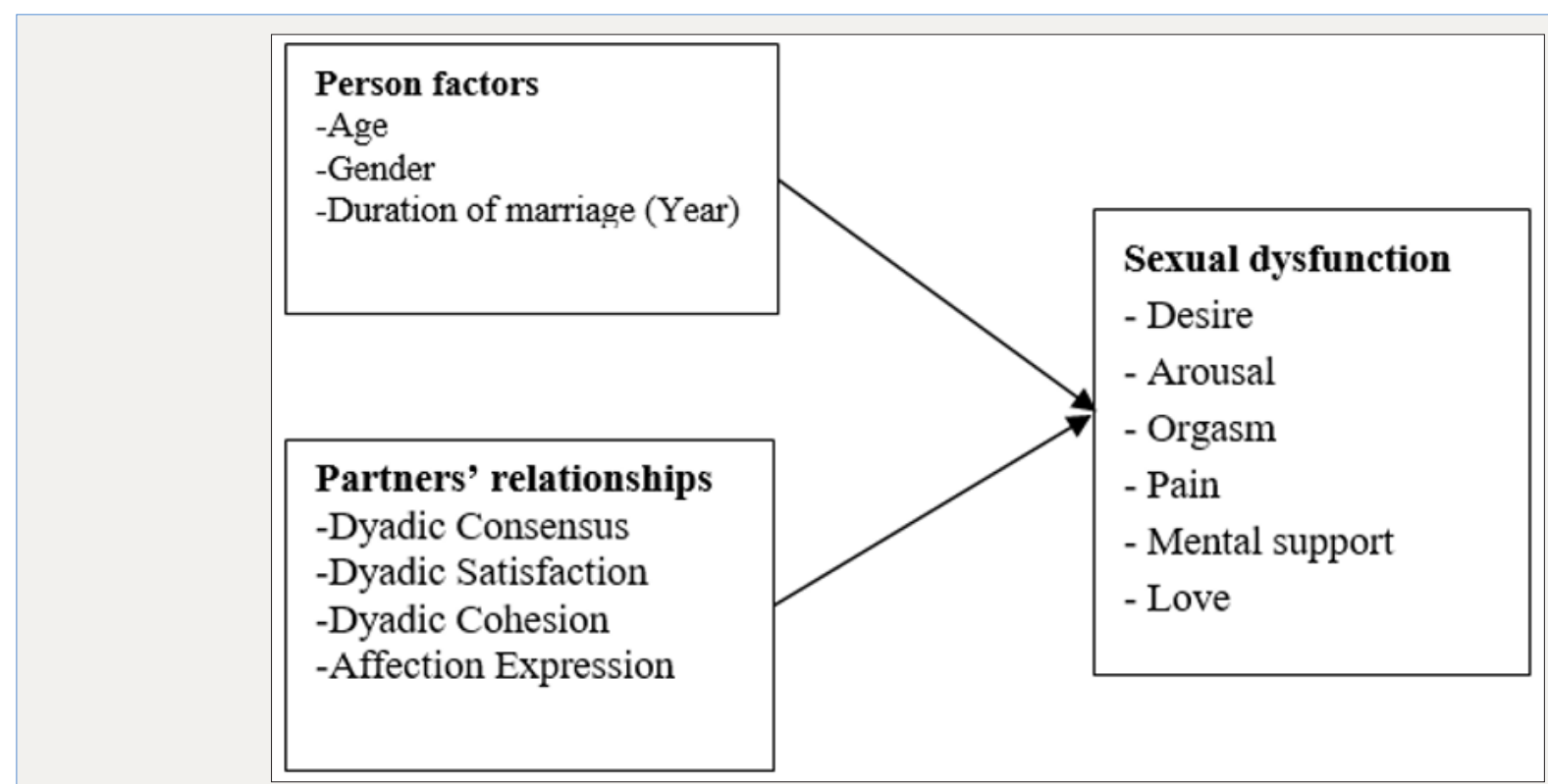

Figure 1: Concept of the study.

\section{Purposes of the study}

Purpose of this study was to examine the sexual dysfunction and factors predicting sexual dysfunction in Thai cancer patients.

\section{Material and Method}

Participants in this study included 55 females with breast cancer and 55 males with cancer at prostate, bladder, and colon. All of them were completed treatment for 6 months- 2 years from a university hospital, and 2 cancer hospitals during March-June 2013. Sample sizes were determined by using Cohen's table [22]. Then, the power 0.80 , alpha 0.05 , with 7 variables, and medium correlation $(\mathrm{r}=0.3)$ from previous study $[14,15]$ were utilized to employ the sample sizes. One hundred and two participants with $10 \%$ attrition rate were 110 .

\section{Instruments}

There were three instruments used as followed: (1) Personal information and disease related treatment questionnaires; (2) Dyadic Adjustment Scale Adjusted Thai Version by Kasemkitawatana (1993); and (3) the Sexual Health Dysfunction questionnaires developed by Kumdaeng [2]. Instruments details were described as followed:

Personal information and disease related treatment questionnaires was developed from literature review. This instrument included personal data, diseases and treatment such as types, stages of cancer and its treatment, time after treatment, and underlying disease. Dyadic Adjustment Scale was first introduced by Spanier [1], and Kasemkitawatana (1993) later translated and adjusted into Thai and Thai context. There were four subscales assessing dyadic adjustment; dyadic consensus, dyadic satisfaction, dyadic cohesion, and affectional expression. There were 28 items with 5 level of likert-scale. The score in each item ranked from 0 (always disagree) to 5 (always agree). There were four components: dyadic consensus 13 items, dyadic satisfaction 7 items, dyadic cohesion 5 items, and affectional expression 3 items. The interpretation of summed score of each subscale were interpreted (higher score means higher dyadic adjustment). Three oncology nurses' experts validated content and the content validity index (CVI) was 0.90. The reliability of the instrument with Cronbach's Alpha Coefficient was 0.89 .

The sexual health dysfunction questionnaires were developed by Kumdaeng [2]. The original instrument was used to assess sexual dysfunction in gynecology cancer patients. It included six subscales of sexual dysfunction; desire, arousal, orgasm, pain, mental support, and love. This instrument was modified to fit with cancer patients in general both male and female. There were 38 items with 17 positive perceptions and 21 negative perceptions. Each item consisted of 4 level of likert-scale ranked from 0 (no at all) to 3 (have problems all the time). There were 6 components: desire 10 items, arousal 5 items, orgasm 5 items, pain (discomfort) 
3 items, mental (psychosocial) 9 items, and relations and love 6 items. The interpretation of summed score of each subscale were interpreted (higher score means higher sexual dysfunction). This instrument was validated content by three oncology nurses' experts, and the content validity index (CVI) was 0.90 . The reliability of the instrument with Cronbach's Alpha Coefficient was 0.91.

\section{Protection of human rights}

For protection of human rights, investigator submitted the study to the Committee on Human Subjects from one university-affiliated hospital and 2 cancer hospitals before collecting data. Then, data collection began after the study was approved by these committees. These procedures were followed the Helsinki Declaration of 1975, as a revised in 2000, ID 08-60-61, with number MURA 2017/601.

\section{Data analysis}

Data were analyzed by Statistical Package for Social Sciences (SPSS) for window version 21 by using the statistics as followed;

A. Personal information and disease related treatment in Thai cancer patients, such as age and duration of marriage, were analyzed by mean and standard deviation. Meanwhile, gender, educational level, types, stages of cancer, and its treatment, income were analyzed by frequency and percent.

B. The overall and 6 subscales of sexual dysfunction which were desire, arousal, orgasm, pain, mental support, and relationship and love were analyzed by mean and standard deviation.
C. Factors predict the sexual dysfunction in Thai cancer patients were analyzed by multiple regression using stepwise technique. Multiple regression analysis used 7 independent variables; age (years), gender (male $=0$ and female $=1$ ), duration of marriage (years), dyadic consensus (score 0-65), dyadic satisfaction (score 0-35), dyadic cohesion (score 0-25), and affectional expression (score $0-15$ ) to predict one dependent variable (sexual dysfunction), with the significant level at 0.05 . Then the multiple regression equation of this study should be

$\beta=\beta_{0}+\beta_{1}$ (Age) $+\beta_{2}$ (Gender) $+\beta_{3}$ (Duration of Marriage $)+\beta_{4}$ (Dyadic Consensus) $+\beta_{5}$ (Dyadic Satisfaction $)+\beta_{6}$ (Dyadic Cohesion) $+\beta_{7}$ (Affectional Expression)

\section{Result}

Results revealed the average age of Thai cancer patients after treatment was 48.63 years $(S D=6.68)$, with the possible range between 30-60. Duration mean of marriage was 21.42 years ( $S D=9.05)$, with the possible range between 1-42. Majority of Thai cancer patients were Buddhism (98.20\%). Half of them (50.91\%) had primary education as their current highest education level. Nearly half of them (43.63\%) had low income which was less than $10,000 \mathrm{baht} / \mathrm{month}$. Majority of them had no comorbid diseases (84.50\%). Data information was shown in Table 1. Results in Table 2 showed that half of them was breast cancer (50\%), followed by cancer of rectum or colon (26.40\%). Majority of cancer patients were stage II A and B (73.64\%). Nearly half of them (44.55\%) received operation and chemotherapy.

Table 1: Personal characteristic of Thai patients with cancer $(n=110)$.

\begin{tabular}{|c|c|c|}
\hline Personal Characteristic & $\mathbf{n}$ & $\%$ \\
\hline \multicolumn{3}{|l|}{ Age $($ years) mean $=48.63, \mathrm{SD}=6.68, \min -\mathrm{max}=30-60$} \\
\hline $30-40$ & 16 & 14.54 \\
\hline $41-50$ & 48 & 43.64 \\
\hline $51-60$ & 46 & 41.82 \\
\hline \multicolumn{3}{|c|}{ Duration of Marriage (years) mean $=21.42, \mathrm{SD}=9.05, \min -\max =1-42$} \\
\hline$<10$ & 11 & 10.00 \\
\hline $10-20$ & 43 & 39.09 \\
\hline $21-30$ & 42 & 38.18 \\
\hline$>30$ & 14 & 12.73 \\
\hline \multicolumn{3}{|l|}{ Gender } \\
\hline Male & 55 & 50.00 \\
\hline Female & 55 & 50.00 \\
\hline \multicolumn{3}{|l|}{ Religion } \\
\hline Buddhism & 108 & 98.20 \\
\hline Christian & 2 & 1.80 \\
\hline \multicolumn{3}{|l|}{ Education } \\
\hline Primary & 56 & 50.91 \\
\hline Lower secondary & 18 & 16.36 \\
\hline Upper secondary & 8 & 7.28 \\
\hline Certificate/Diploma & 18 & 16.36 \\
\hline
\end{tabular}




\begin{tabular}{|c|c|c|}
\hline \multicolumn{3}{|l|}{ Education } \\
\hline Bachelor & 9 & 8.18 \\
\hline Post graduate & 1 & 0.91 \\
\hline \multicolumn{3}{|l|}{ Income (baht/month) } \\
\hline$<10,000$ & 48 & 43.63 \\
\hline $10,000-30,000$ & 38 & 34.55 \\
\hline $30,000-50,000$ & 15 & 13.64 \\
\hline$>50,000$ & 9 & 8.18 \\
\hline \multicolumn{3}{|l|}{ Comorbid Disease } \\
\hline No & 93 & 84.50 \\
\hline Yes & 17 & 15.50 \\
\hline
\end{tabular}

Table 2: Description of types of cancer and treatments $(n=110)$.

\begin{tabular}{|c|c|c|}
\hline Types of Cancer and Treatments & $\mathbf{n}$ & $\%$ \\
\hline \multicolumn{3}{|l|}{ Types of Cancer } \\
\hline Breast & 55 & 50.00 \\
\hline Rectum/Colon & 29 & 26.40 \\
\hline Prostate & 14 & 12.70 \\
\hline Bladder & 12 & 10.90 \\
\hline \multicolumn{3}{|l|}{ Stages of Cancer } \\
\hline Stage I & 5 & 4.55 \\
\hline Stage II A & 44 & 40.00 \\
\hline Stage II B & 37 & 33.64 \\
\hline Stage III A & 20 & 18.18 \\
\hline Stage III B & 4 & 3.63 \\
\hline \multicolumn{3}{|l|}{ Treatments received } \\
\hline Operation & 27 & 24.55 \\
\hline Operation and Chemotherapy & 49 & 44.55 \\
\hline Operation and Radiotherapy & 6 & 5.45 \\
\hline Operation, Chemotherapy and Radiotherapy & 28 & 25.45 \\
\hline
\end{tabular}

Table 3: Characteristic of sexual dysfunction and partner's relationship in Thai patients with cancer $(\mathrm{n}=110)$.

\begin{tabular}{|c|c|c|c|c|}
\hline Variables & Possible range & Actual range & Mean & SD \\
\hline Sexual Dysfunction (total) & $0-114$ & $11-89$ & 36.65 & 13.83 \\
\hline Desire & $0-30$ & $1-25$ & 11.87 & 4.90 \\
\hline Arousal & $0-15$ & $1-13$ & 5.68 & 2.44 \\
\hline Orgasm & $0-15$ & $0-15$ & 6.00 & 3.06 \\
\hline Pain & $0-9$ & $0-9$ & 8.41 & 1.70 \\
\hline Mental health & $0-27$ & $0-24$ & 3.29 & 4.18 \\
\hline Love & $0-18$ & $0-17$ & 109.29 & 3.23 \\
\hline Dyadic Adjustment (total) & $0-140$ & $63-140$ & 53.61 & 13.94 \\
\hline Dyadic Consensus & $0-65$ & $36-65$ & 28.33 & 6.28 \\
\hline Dyadic Satisfaction & $0-35$ & $15-35$ & 16.47 & 4.72 \\
\hline Dyadic Cohesion & $0-25$ & $7-25$ & 10.88 & 4.17 \\
\hline Affectional Expression & $0-15$ & $4-15$ & \\
\hline
\end{tabular}

Results in Table 3 showed that the average of total sexual dysfunction was 36.65 ( $S D=13.83$ ), possible range of $0-114$. It meant that Thai cancer patients had low sexual dysfunction both in total scores and 6 subscales. However, the average of total partner's relationship was 109.29 ( $\mathrm{SD}=13.94)$, possible range of 0 -140. It meant that Thai cancer patients had high partner's relationship both in total scores and 4 subscales. Details has been shown in Table 3. 
Result demonstrated that factors predict the sexual dysfunction in Thai cancer patients after treatment were affectional expression ( $b=-2.075, p<0.01)$, dyadic consensus (b-.517, $p=0.014)$, and gender $(b=5.281, p=0.034)$. These variables could predict the sense of coherence $23.4 \%$. Meanwhile, age, duration of marriage, and other two subscales of dyadic adjustment could

Table 4: Factors predict sexual dysfunction in Thai patients with cancer after treatments $(n=110)$ Constant $=84.291, R$ square $=23.4 \%$, SE: Standard Error

\begin{tabular}{|c|c|c|c|c|c|c|c|}
\hline Variables & b & SE b & Beta & R & R2 & F change & Sig. \\
\hline $\begin{array}{c}\text { Affectional } \\
\text { Expression }\end{array}$ & -2.075 & 0.549 & -0.361 & 0.404 & 0.163 & 21.046 & 0.000 \\
\hline $\begin{array}{c}\text { Dyadic } \\
\text { Consensus }\end{array}$ & -0.517 & 0.207 & -0.235 & 0.447 & 0.200 & 4.952 & 0.014 \\
\hline $\begin{array}{c}\text { Gender (male }= \\
\text {, female = 1) }\end{array}$ & 5.281 & 2.453 & 0.192 & 0.483 & 0.234 & 4.635 & 0.034 \\
\hline
\end{tabular}

\section{Discussion}

Results of this study confirmed that Thai cancer patients had mild to moderate sexual dysfunction in both total and six subscales, however, they had high scores of dyadic adjustments of both total and four subscales. These results were congruent with previous studies $[14,15]$. These previous studies found that marriage adjustment was highly negative correlated with sexual dysfunction, and it also could predict the sexual dysfunction in this study. Since dyadic adjustment consists of dyadic consensus, satisfaction, cohesion, and affectional expression, these relationships help pulling the cancer patients and their partners to adjust their sexual life after cancer treatment together. A good relationship can negatively influence on their sexual dysfunction. This means that, with high degree of dyadic adjustment, they could go through their suffering period of sexual dysfunction after cancer treatments [23]. Healthcare providers should assess the dyadic adjustment and sexual dysfunction of cancer patients and their partners [24] especially female with cancer. In case of their previous low dyadic adjustment, the sexual dysfunction was more likely to occur after cancer treatment. Healthcare providers should develop the specialty clinic such couple-based intervention program to support cancer patients after treatment [25]. Even though there are adequate information and group support from university hospital, it still needs such specialty clinic created for those cancer patients and their partners to support and help them adjusting themselves in the period of post cancer treatment.

Result from this study also demonstrated that gender could predict the sexual dysfunction. This result was congruent with previous study of Junda et al. [17]. However, the study of Hendren and colleagues stated otherwise, male rectal cancer patients had higher sexual dysfunction after surgery [16]. In this study, the result showed that women with breast cancer suffered more from sexual dysfunction than men with prostate, bladder, or colon. The reason explaining more suffering in women is that they loss their feminine organ from cancer and its treatment which directly decrease their desire in sexual relation. In addition, because of conservative mindset of Thai people, women cannot express any sexual related concerns. As a result, Thai female was reluctant to express their not predict the sexual dysfunction in Thai patients of cancer. Then, the multiple regression equation for this study was Sexual Dysfunction $=84.291-2.075$ (Affectional Expression) -0.517 (Dyadic Consensus)+5.281(Gender). Further information was shown in Table 4.

sexual problem to both healthcare providers and their partners Moreover, women are more sensitive than men. The same problem may mentally effects on women more than men. Thus, healthcare providers should assess and relief the sexual dysfunction, focusing on women with cancer.

In case of age and duration of marriage, both could not predict the sexual dysfunction. These results could be explained that the average age was $48.63, \mathrm{SD}=6.68$. The result was congruent with previous studies. Most participants were in the middle age with long duration of marriage (mean=21.42 years, $S D=9.05$ ) and fulfilled with their sexual life experience. Thus, they may not concern much on their sexual dysfunction. In addition, in Thai culture, people who were in middle age with long duration of marriage usually have separately activities and do less dyadic activities together leading to less opportunity to express their love. In addition, majority of the participants were in the same period of age (41-60 years, $85.46 \%$ ) and duration of marriage (10-30 years, $77.27 \%)$. As a result, the scores of sexual dysfunctions were in the same way, it cannot predict the sexual dysfunction in these cancer patients after treatment.

\section{Conclusion}

It can be summarized that gender and two subscales of dyadic adjustment were strong predictors of the sexual dysfunction in Thai cancer patients after treatment. Health care team should promote dyadic adjustment especially in female cancer patients to improve the sexual dysfunction after treatment.

\section{What is already known on this topic?}

The relationship between the dyadic adjustment and sexual dysfunction in both genders of cancer patients has been studied in Thailand with moderate correlation. In addition, there were differences of sexual dysfunction in male and female. The study of factors predicting of the sexual dysfunction in Thai cancer patients is needed to be done.

\section{What this study adds}

This is the first study of factors predicting of the sexual dysfunction in Thai cancer patients in Thailand. It included subscales of dyadic adjustment to explain the sexual dysfunction. 


\section{Acknowledgement}

The authors gratefully thank Faculty of Medicine Ramathibodi Hospital, Mahidol University for funding support and all participants participating in this study.

\section{References}

1. Spanier GB (1976) Measuring dyadic adjustment: New scales for assessing the quality of marriage and similar dyads. J marriage and the Family 38(1): 15-25.

2. Kumdaeng P (2007) Sexual dysfunction and management in women with gynecological cancer after treatment. Cheng Mai University, Thailand.

3. Hughes MK (1996) Sexuality issues: Keeping your cool. Oncol Nurs Forum 23: 1597-1600.

4. Emilee G, Ussher JM, Perz J (2010) Sexuality after breast cancer: A review. Maturitas 66(4): 397-407.

5. Traa MJ, Vries JD, Roukema JA, Den Oudsten BL (2012) Sexual (dys) function and the quality of sexual life in patients with colorectal cancer: a systematic review. Annals of Oncology 23(1): 19-27.

6. O Shaughnessy PK, Ireland C, Pelentsov L, Thomas LA, Esterman A (2013) Impaired sexual function and prostate cancer: a mixed method investigation into the experiences of men and their partners. J Clin Nurs 22(23-24): 3492-502.

7. Dunn MW (2015) Bladder cancer: A focus on sexuality. Clin J Oncol Nurs 19(1): 68-73.

8. Shell JA (2002) Evidence-based practice for symptom management in adults with cancer: Sexual dysfunction. Oncol Nurs Forum 29(1): 53-66.

9. Wang F, Chen F, Huo X, Xu R, Wu L, et al. (2013) A neglected issue on sexual well-being following breast cancer diagnosis and treatment among Chinese women. PLOS one 8(9).

10. http://www.nci.go.th/th/File_download/Nci\%20Cancer\%20Registry/ Hospital-Based\%20NCI\%202012\%20Total.pdf

11. http://www.nci.go.th/th/File_download/Nci\%20Cancer\%20Registry/ HOSPITAL-BASED\%202013.pdf

12. http://www.nci.go.th/th/File_download/Nci\%20Cancer\%20Registry/ HOSPITAL-BASED\%202014.pdf
13. http://www.nci.go.th/th/File_download/Nci\%20Cancer\%20Registry/ HOSPITAL-BASED\%202015.pdf

14. http://www.nci.go.th/th/File_download/Nci\%20Cancer\%20Registry/ Hospital-Based\%20NCI2\%202016\%20Web.pdf

15. Prakobkit K, Junda T, Sumdaengrit B (2014) Marital relationship before surgery, sexual health, and quality of life in women with breast cancer after surgery and their spouses. J Nurs Health Care 32: 14-23.

16. Wattanapansak A, Junda T, Sumdaengrit B (2014) Marital relationships before treatment, sexual health, and quality of life in male patients with prostate, bladder, colon, and rectum cancer after treatment and their partners. J Nurs Health Care 32: 103-113.

17. Hendren SK, O Conner BI, Liu M, Asano T, Cohen Z, (2005) Prevalence of male and female sexual dysfunction is high following surgery for rectal cancer. Annals of Surgery 242(2): 212-223.

18. Junda T, Sumdaengrit B (2018) Gender and sexual dysfunction in Thai cancer patients: A comparative study. J Med Assoc Thai.

19. Den Oudsten BL, Van Heck GL, Vander Steeg AFW, Roukema JA, De Vries J (2010) Clinical factors are not the best predictors of quality of sexual life and sexual functioning in women with early stage breast cancer. Psycho-Oncology 19(6): 646-656.

20. Greendale GA, Petersen L, Zibecchi L, Ganz PA (2001) Factors related to sexual function in postmenopausal women with a history of breast cancer. Menopause 8(2): 111-119.

21. Quintard B, Constant A, Lakdja F, Labeyrie Lagardère H (2014) Factors predicting sexual functioning in patients 3 months after surgical procedures for breast cancer: The role of the Sense of Coherence. Euro J Oncol Nurs 18(1): 41-45.

22. Schroder J, Schmiedeberg C (2015) Effects of relationship duration, cohabitation, and marriage on the frequency of intercourse in couples: Findings from German panel data. Social Science Research 52: 72-82.

23. Cohen J (1992) A power primer. Psychological Bulletin 112(1): 155159.

24. Krebs LU (2008) Sexual assessment in cancer care: Concepts, methods, and strategies for success. Sem Oncol Nurs 24(2): 80-90.

25. Jonsdottir JI, Jonsdottir H, Klinke ME (2018) A systematic review of characteristics of couple-based intervention studies addressing sexuality following cancer. J Adv Nurs 74(4): 760-773.
Creative Commons Attribution 4.0 International License

For possible submissions Click Here

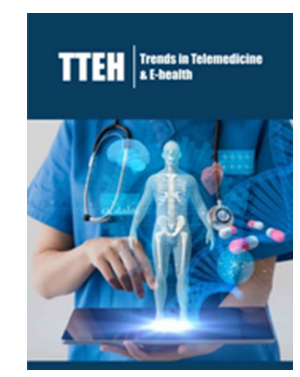

Trends in Telemedicine \& E-health

\section{Benefits of Publishing with us}

- High-level peer review and editorial services

- Freely accessible online immediately upon publication

- Authors retain the copyright to their work

- Licensing it under a Creative Commons license

- Visibility through different online platforms 